\title{
A New Microhardness Testing Method on Grating Films
}

\author{
Haitao Ma $\mathbb{D}^{1}{ }^{1}$ Yi Jin, ${ }^{2}$ and Jirigalantu $\mathbb{D}^{3,4}$ \\ ${ }^{1}$ School of electrical and electronic engineering, changchun university of technology, Changchun 130012, Jilin, China \\ ${ }^{2}$ Department of Precision Machinery and Precision Instrumentation, School of Engineering Science, \\ University of Science and Technology of China, Hefei 230027, Anhui, China \\ ${ }^{3}$ Changchun Institute of Optics, Fine Mechanics and Physics, Chinese Academy of Sciences, Changchun 130033, Jilin, China \\ ${ }^{4}$ Yanshan University, Qinhuangdao 066004, Hebei, China \\ Correspondence should be addressed to Jirigalantu; jiri5998@163.com
}

Received 8 July 2019; Revised 24 November 2019; Accepted 16 December 2019; Published 17 February 2020

Academic Editor: Mitsuhiro Okayasu

Copyright (c) 2020 Haitao Ma et al. This is an open access article distributed under the Creative Commons Attribution License, which permits unrestricted use, distribution, and reproduction in any medium, provided the original work is properly cited.

The manufacturing process of large-area, high-precision gratings is a very complicated and time-consuming process. The hardness testing of grating films is an important step in the entire process. In order to simplify the manufacturing process of gratings, we have proposed a new method for testing microhardness based on tool edge indentation. Also, it unified tool adjustment and microhardness testing steps in the grating manufacturing process. First, a mathematical model of the relationship between tool load and indentation contour length is established. The model parameters were then modified using tool indentation experiments with different loads. When measured with a nanoindenter, the average hardness of the grating film was $447 \mathrm{MPa}$. The hardness value of the grating film obtained by our proposed method is almost the same as that measured by the nanoindenter, and the maximum deviation is about $2.2 \%$ of the average hardness value. The experimental results show that our proposed method can replace the microhardness test method of using a nanoindenter. Therefore, the disadvantages of using a nanoindenter to test the hardness of a grating film are avoided, such as the limited sample size, the sensitivity of the indenter to the roughness of the film and the depth of the indentation, and the accuracy of film testing, and the efficiency of grating ruling can be improved.

\section{Introduction}

In optics, a diffraction grating is an optical component with a periodic structure that splits and diffracts light into several beams travelling in different directions, and its cross section usually shows a serrated, sinusoidal, or rectangular array. Gratings have many applications in a variety of spectrometers [1] and, especially, have received great attention for their applications in the selection of laser frequency and bandwidth compression area [2-5]. As the core component of high-precision spectral instruments [6], gratings are widely used in military, astronomy, alternative energy, biochemical analysis [7], and other fields [8]. Currently, large-area, high-precision gratings are an area of active research in the grating ruling field. Such gratings have a width of more than $400 \mathrm{~mm}$ [9] and a groove density of more than $3600 \mathrm{~g} / \mathrm{mm}$. The large area can help gratings achieve excellent light-gathering and resolving power, whereas the high-precision ruling can endow instruments with an excellent signal-to-noise ratio and wave front quality $[10,11]$.

To date, the mechanical ruling method is the main method to manufacture large-area, high-precision gratings $[12,13]$. A diamond tool is used to extrude and polish the metal film on a grating substrate, to generate periodic v-shaped grooves on the film surfaces without forming chips or scrapes during the entire process. The film is usually made of pure aluminum or gold through an electron beam evaporation process, which is soft and exhibits good plasticity [14]. The mechanical ruling process is shown in Figure 1. The microstructure on an aluminum film of gratings can be observed using an electron microscope, as shown in Figure 2.

The grating ruling process is complicated and involves many steps, such as film hardness testing, tool aligning and adjusting (according to load versus tool indentation profile), trial ruling and load optimizing, and formal ruling. Among 


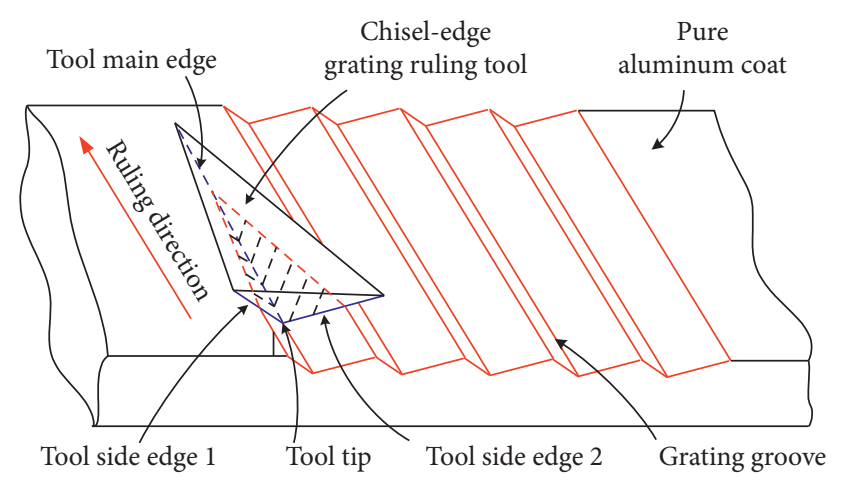

FIGURE 1: Grating ruling diagram.

these steps, the tool adjusting and load optimizing process are the two key steps. During the grating ruling process, to attain the best extruded and polished grating blaze plane and to ensure a regular elastic-plastic uplift in the lateral part of the triangular groove, the consistency of the grating ruling tool main edge and the ruling direction should be strictly guaranteed, and the appropriate load should be optimized according to the hardness of the aluminum film. Thus, accurately evaluating the aluminum film hardness is very important. When the aluminum film on the grating substrates is too hard, the extruding process becomes difficult and the polishing effect becomes poor. On the other hand, when the aluminum film on the grating substrates is too soft, the extruding process becomes easy and the polishing effect becomes fine, but the groove shape is not easy to form accurately. The optimum value of the aluminum film hardness always depends on the experience of the grating ruling. So, during the grating ruling process, the hardness can be used as an important test standard for the quality of grating aluminum film.

In previous studies of the grating ruling process, the features of tool indentation profile on the film were used to roughly judge the hardness status of the aluminum film of the gratings, but it could not quantify the hardness value of the film. Currently, nanoindenters are used to test the hardness of small grating samples (which are coated film with a large grating at the same time), and that represents the hardness of larger grating aluminum films to some extent. However, nanoindenters exhibit many shortcomings in testing grating films. For example, aluminum films for large gratings cannot be directly tested by nanoindenters because of their limited size, and the macroscopic inhomogeneity of thick film easily leads to inconsistency of measurement results. In addition, the nanoindenter is very sensitive to film roughness, and the depth of the indentation region is limited.

As mentioned above, tool indentation is a key step in the grating ruling process. After tool indentation, the projection line of the main edge of the indentation can be used to judge the consistency of the ruling direction, and the length of the line can not only be used to estimate the pitch angle of the ruling tool but also can be used to roughly determine the hardness state and elastic-plastic properties of the grating film.

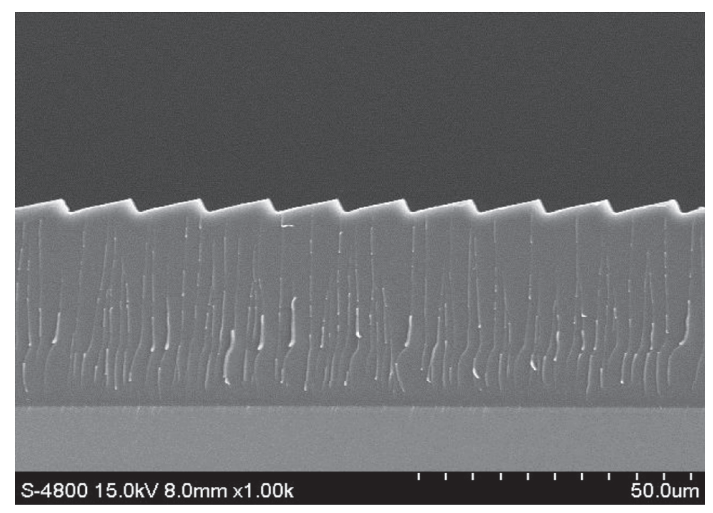

FIgURe 2: Microstructure of a grating aluminum film.

If the hardness of an aluminum film can be accurately determined by measuring the tool indentation length, it will provide many advantages to the grating ruling process. First, the traditional nanoindentation head and the ruling tool edge can be combined. Second, test of hardness and tool indentation processes can be unified. Third, the hardness testing of grating films can be combined with the grating ruling process. If this hardness testing method works well, it would greatly increase the efficiency of the grating ruling process and eliminate the cost of manufacturing test samples for large grating film and the hardness testing work using a nanoindenter. In addition, since the contact area between the ruling tool edge and the aluminum film is large, the hardness of the aluminum film calculated by using the length of the tool indentation is not sensitive to the roughness of the aluminum film. This can effectively improve the accuracy and reliability of the hardness test on the aluminum film. So, the hardness of the grating film as expressed by the length of the tool indentation is of great value to the grating ruling process. This paper proposed a new microhardness testing method for a grating film based on grating ruling tool indentation, and this method has important application value in improving the processing efficiency of the ruling gratings. The grating microhardness testing method according to load versus tool indentation profile is mathematically modeled, and the index of the aluminum film hardness model is determined by theoretical and experimental analysis.

\section{Theoretical Background}

The structure of the grating ruling tool is shown in Figure 3 [15]. The cross section of the grating ruling tool is an asymmetrical "V" shape, and its main parameters include the orientation angle $(D)$, nonorientation angle $(F)$, and back oblique angle $(H)$.

In Figure 3, the grating ruling directions are specified in the $X$-axis directions on the coordinate system, and when the grating is ruled, the main edge of the ruling tool lifts at a small angle in the $X-Z$ plane, which is called the pitch angle $(E)$. Beside the main edge are the two sides of the tool edge, respectively, named the orientation plane and the nonorientation plane. Also, the two planes intersect with the 


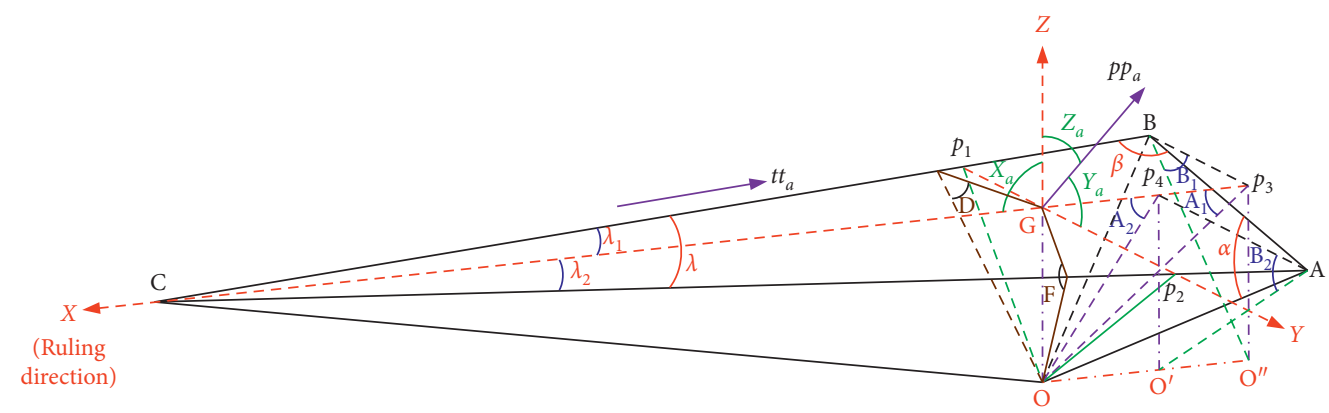

FIGURE 3: Structure of the grating ruling tool [15].

back oblique plane and form two side edges, which are the orientation side edge and the nonorientation side edge.

Based on our previous study [15], a set of geometry formulas can be developed, and using MATLAB software, the parametric geometric model can be calculated for the grating ruling tool. When the grating ruling tool is ruled on the aluminum film, it is subjected to deformation resistance force from the aluminum film on the $X, Y$, and $Z$ directions. When the tool edge is pressed into the aluminum film under a certain load and becomes static, the load on the grating ruling tool and the deformation resistance force from the aluminum film in the $Z$ direction are balanced. The algebraic sum of the deformation resistances force from the $X$ and $Y$ directions is always zero because they are not related to the load and thus can be ignored. We can define the positive stress of the orientation plane as $p p_{a}$ and the angle between the positive stress $p p_{a}$ and the $Z$ axis as $z_{a}$. Similarly, we can define the positive stress of the nonorientation plane as $p p_{b}$ and set angle between the positive stress $p p_{b}$ and the $Z$ axis as $z_{b}$.

According to the set of Tabor, when Poisson's ratio of the material is 0.3 , the maximum contact stress $p p$ that causes plastic deformation is directly related to the hardness of soft materials. Namely, for $p p=0.6 H_{\mathrm{n}}$ [16], the actual hardness $H_{\mathrm{n}}$ of the soft aluminum film can be obtained by testing with a nanoindenter. Therefore, the relationship of stress and $H_{\mathrm{n}}$ can be determined as

$$
p p_{a}=p p_{b}=p p_{c}=p p=0.6 \times H_{\mathrm{n}} .
$$

According to Liu's research [14], the coefficient of 0.6 in equation (1) is used for calculating the hardness of soft metal materials. In our research, this coefficient value was used to establish the tool indentation model. However, the grating film material is a soft aluminum film with a thickness on the micron or submicron scale, and a hard optical glass basement is present under the film, which leads to a basement effect on testing microhardness. Thus, the actual coefficient value in equation (1) will be revised through the tool indentation experiment.

In Figure 3, we can see that $z_{a}=D, z_{b}=F$, and $z_{c}=H$, and we can define $S_{a z}$ as the projection area of area $S_{a}$ on the $X-Y$ plane. Similarly, we can define $S_{b z}$ as the projection area of area $S_{b}$ on the $X-Y$ plane and define $S_{c z}$ as the projection area of area $S_{c}$ on the $X-Y$ plane, which can be expressed as

$$
S_{a z}=S_{a} \cos \left(z_{a}\right) \text {. }
$$

We can define $p z_{a}, p z_{b}$, and $p z_{c}$ as the separated forces of the unit normal stress on the $Z$ axis for each plane of the grating ruling tool, which can be expressed as

$$
p z_{a}=p p \cos \left(z_{a}\right) \text {. }
$$

We can define $P z_{a}, P z_{b}$, and $P z_{c}$ as the separated forces of the total normal stress on the $Z$-axis for each plane of the grating ruling tool, which can be expressed as

$$
P z_{a}=p z_{a} S_{a} .
$$

The sum of the total positive stress in the orientation plane, nonorientation plane, and the back oblique plane applied on the $Z$-axis is

$$
P z=P z_{a}+P z_{b}+P z_{c}
$$

Based on the abovementioned formulas and using the program developed by MATLAB software, we performed the calculations of concerning parameters and drew a set of curves that $P z, P z / a$, and $P z / a^{2}$ change with the length of the tool indentation, as shown in Figure 4. The hardness value of the soft aluminum film in the program is $H_{\mathrm{n}}=447 \mathrm{MPa}$, which is the average of the hardness data obtained using the nanoindenter, and this value can be used as the hardness value of the soft aluminum film in our model. Figure 4(a) shows that the $Z$-direction force increases with the length of the ruling tool indentation and the increasing trend shows a quadratic curve. The ratio of the $Z$-direction force to the length of the ruling tool indentation increases linearly with the increasing length of the ruling tool indentation, as shown in Figure 4(b). Also, the ratio of the $Z$-direction force to the length square of the ruling tool indentation is a constant, as shown in Figure 4(c).

Therefore, according to the calculation results in Figure 4 and the Knoop hardness testing method, the theoretical formula for the aluminum film hardness can be obtained as

$$
H_{G}=C \frac{P}{l^{2}},
$$

where the hardness index is $C=447 / 11.314=39.5086$. By substituting this index into equation (6), we obtain

$$
H_{G}=39.5086 \frac{P}{l^{2}} \text {. }
$$




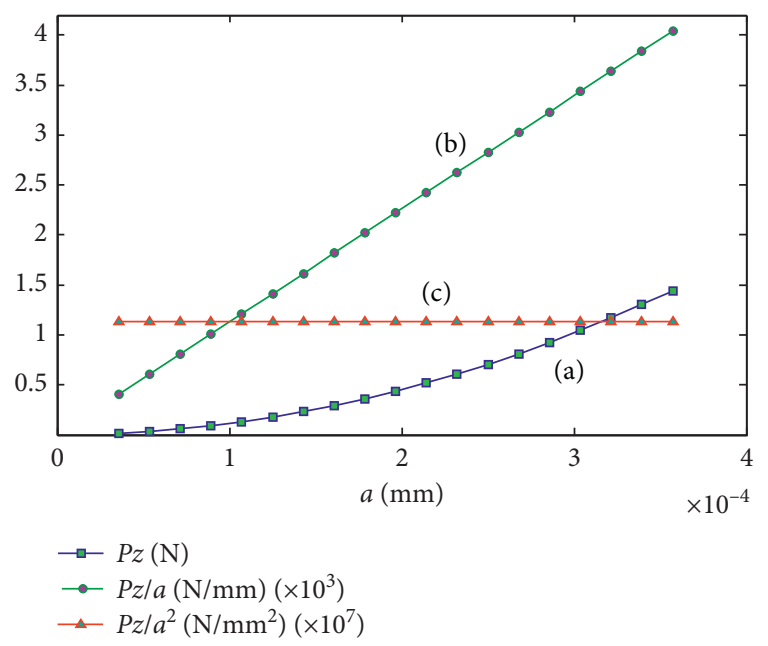

Figure 4: Curves wherein $P z, P z / a$, and $P z / a^{2}$ change with the length of the tool indentation.

\section{Experimental Techniques}

To verify the accuracy of the developed mathematical model, a grating ruling tool was manufactured according to the theoretically calculated parameters. The tool material is fine octahedral natural diamond, and the tool edge is polished and finished by the Ultra precision natural diamond lapping machine. Then, the tool is installed on the CIOMP-5 grating ruling machine at the Changchun Optical Precision Machinery and Physics Institute, as shown in Figure 5.

The grating film is a $11 \mu \mathrm{m}$-thick pure aluminum film, its uniformity of thickness was less than $1 \%$, and the surface roughness was about $R_{\mathrm{a}}=20 \mathrm{~nm}$. The film was deposited on K9 substrates by the electron-beam evaporation process, with the hardness of $447 \mathrm{MPa}$ tested by an Agilent, Nano Indenter G200 [17].

The load is applied on the grating ruling tool using weights, and seven weights are prepared starting from 5 grams to 35 grams, with an incremental step of 5 grams. The cam mechanism is used to realize the lifting and lowering functions, namely loading and unloading functions, and the loading and unloading rate is about $1 \mathrm{~mm} / \mathrm{s}$. The length of the tool indentation is obtained under a $\times 1000$ optical microscope with an accuracy of 0.1 microns.

Then, several indentation experiments were performed on grating aluminum films. The load on the grating tool was increased from 5 grams to 35 grams with an incremental step of $5 \mathrm{~g}$. In each experiment, an optical microscope was used to observe the tool profile and measure the length of indentation, according to which the tool indentation profile was drawn.

\section{Results and Discussion}

The shape of the tool indentation profile under different loads was compared and analyzed. The profile of the tool indentation was observed to expand regularly as the load increased from 5 grams to 35 grams, and the length of the tool indentation was also regularly increased within a certain range, as shown in Figure 6.

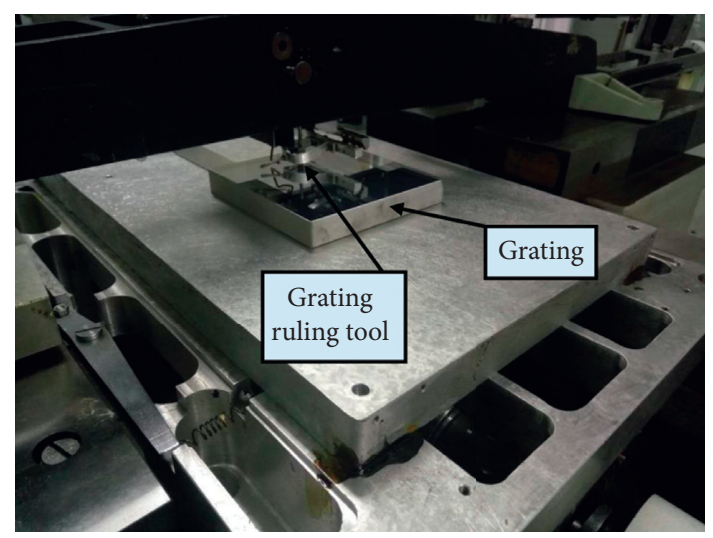

Figure 5: CIOMP-5 grating ruling machine.

Table 1 shows how the parameters of the tool indentation vary with the increasing load. The ratio $P / l^{2}$ of the load $P$ to the length square $l^{2}$ changed at approximately 13.95 , and the standard deviation was within 0.17 . According to the load on the tool, the length of the tool indentation, and the ratio of the load to length square, combined with the hardness of the aluminum film measured with the nanoindenter, the value of the hardness index $\mathrm{C}$ could be calculated using equation (6).

According to the data in Table 1, the average hardness index $\mathrm{C}$ based on the microhardness test formula for the tool indentation was 32.06, with a standard deviation of 0.57 , revealing an accuracy of $3 \%$. Compared with the hardness index obtained from the theoretical calculation in Section 2, the relative error was $23.23 \%$. As mentioned above, this deviation is caused by the coefficient value in equation (6), which is not suitable for a grating film since under the film presents a hard optical glass basement, and it lead to a basement effect. In the experimental study, the coefficient was revised to 0.76 . Since the material of the grating aluminum film is fixed, the experimental hardness index can also be used to replace the simulation value in Section 2 . Therefore, the average hardness index $C$ was input into equation (6) that is developed on the relationship between the load on the tool and the length of the tool indentation, and the final microhardness model for grating aluminum films was obtained as follows:

$$
H_{G}=C \frac{P}{l^{2}}=32.06 \frac{P}{l^{2}} \text {. }
$$

According to equation (8), the maximum and minimum hardness of the aluminum film are $456.855 \mathrm{MPa}$ and $440.825 \mathrm{MPa}$, respectively. The difference between the two values is $16.03 \mathrm{MPa}$, and these values are very close to the average hardness value of $447 \mathrm{MPa}$ measured by the nanoindenter, and the maximum deviation between the two test results was below $2.2 \%$ of the average hardness. These results demonstrate the accuracy and validity of the proposed microhardness model about aluminum films of gratings.

In addition, when testing the aluminum film by our method, the hardness versus load curve may show a tendency same as that of the Vickers hardness and Knoop hardness test methods, that the hardness decreases rapidly as 


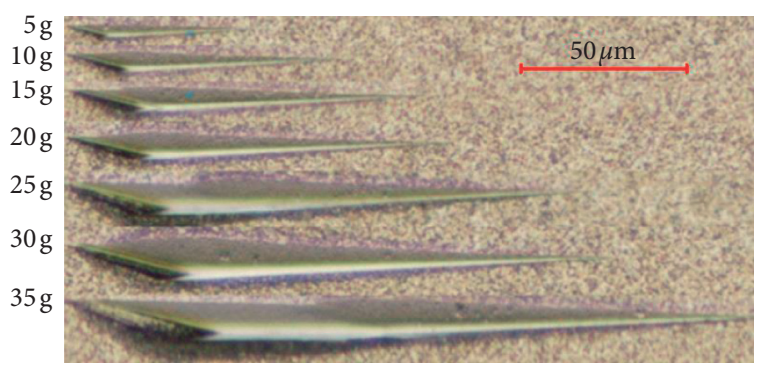

FIGURE 6: Variations in the tool indentation length versus the tool load.

TABLE 1: Variations in the parameters of the tool indentation with tool load.

\begin{tabular}{lcccc}
\hline$P(\mathrm{~g})$ & $l(\mu \mathrm{m})$ & $P / l^{2}(\mathrm{MPa})$ & $C$ & $H_{\text {Nano }}(\mathrm{MPa})$ \\
\hline 5 & 57.90 & 13.75 & 32.51 & \\
10 & 83.60 & 14.02 & 31.88 & \\
15 & 103.30 & 13.78 & 32.44 & \\
20 & 117.30 & 14.25 & 31.37 & 447 \\
25 & 133.10 & 13.83 & 32.32 & \\
30 & 145.40 & 13.91 & 32.14 & \\
35 & 156.10 & 14.08 & 31.75 & \\
Mean & & 13.95 & 32.06 & \\
Std. dev. & & 0.1676 & 0.57 & \\
$\%$ cov. & & 1.20 & 1.78 & \\
\hline
\end{tabular}

the applied load increases, and then it attains saturation for higher loads $[18,19]$. However, in general, the load range used on testing grating aluminum film hardness is approximately 10 to 30 grams, and stable hardness test results are obtained in the range of 5 to 35 grams. Therefore, this paper does not give great attention to study the hardness testing effect on the shallow layer of the grating aluminum film.

\section{Conclusion}

In this paper, a new method for measuring the hardness of soft films of gratings was proposed, and its theoretical calculation model was established. Then, the tool indentation experiment was successfully prepared and conducted on the grating ruling machine. The experimental results indicated that the addition of grating basement has some effect on the determination of hardness index $(C=39.508)$, and after revising the coefficient of 0.6 in equation (1) to 0.76 , the calculated hardness index was well fitted to the results $(C=32.06)$ obtained by tool indentation experiment. Also, based on the experimentally determined new formula, the hardness value of grating aluminum film was obtained, and it was varied between $456.855 \mathrm{MPa}$ to $440.825 \mathrm{MPa}$ approximately; the value was very close to the average hardness value of $447 \mathrm{MPa}$ measured by the nanoindenter, the maximum deviation was below $2.2 \%$ of the average hardness, and it sounds accurate and reliable. These results indicated that the new proposed method may replace the traditional nanointender hardness test on grating film and also is an interesting new way to unify the hardness testing process with the tool adjusting process in the grating ruling process, which not only saves time but also improves measurement reliability.

\section{Data Availability}

All the data used in the manuscript can be obtained by experiments.

\section{Conflicts of Interest}

The authors declare that they have no conflicts of interest.

\section{Acknowledgments}

This study was supported by the National Key R\&D Program of China (2018YFA0703400) and the Jilin Province Science and Technology Innovation Project of China (grant 20190201021JC).

\section{References}

[1] J. Song, L. Chen, and B. Li, "A fast simulation method of silicon nanophotonic echelle gratings and its applications in the design of on-chip spectrometers," Progress in Electromagnetics Research, vol. 141, pp. 369-382, 2013.

[2] Z. Li, S. Li, C. Wang et al., "Stable and near Fourier-transformlimit 30fs pulse compression with a tiled grating compressor scheme," Optics Express, vol. 23, no. 26, pp. 33386-33395, 2015.

[3] X. Wang, X. Wei, Y. Hu et al., "Chirped-pulse amplification system based on chirp reversal and near-field spatial reversal with common tiled grating pair as stretcher and compressor," Applied Optics, vol. 51, no. 23, pp. 5627-5632, 2012.

[4] R. Kammel, R. Ackermann, J. Thomas et al., "Enhancing precision in fs-laser material processing by simultaneous spatial and temporal focusing," Light: Science \& Applications, vol. 3, no. 5, p. e169, 2014.

[5] J. Qiao, A. W. Schmid, L. J. Waxer et al., "In situ detection and analysis of laser-induced damage on a $15-\mathrm{m}$ multilayer-dielectric grating compressor for high-energy, petawatt-class laser systems," Optics Express, vol. 18, no. 10, pp. 10423-10431, 2010.

[6] C.-H. Chang, Y. Zhao, R. K. Heilmann, and M. L. Schattenburg, "Fabrication of $50 \mathrm{~nm}$ period gratings with multilevel interference lithography," Optics Letters, vol. 33, no. 14, pp. 1572-1573, 2008.

[7] N. Bonod and J. Neauport, "Diffraction gratings: from principles to applications in high-intensity lasers," Advances in Optics and Photonics, vol. 8, no. 1, pp. 1-44, 2016.

[8] M. P. Wood and J. E. Lawler, "Aberration-corrected echelle spectrometer for measuring ultraviolet branching fractions of iron-group ions," Applied Optics, vol. 51, no. 35, pp. 84078412, 2012.

[9] G. R. Harrison, S. W. Thompson, H. Kazukonis, and J. R. Connell, "750-mm ruling engine producing large gratings and echelles," Journal of the Optical Society of America, vol. 62, no. 6, pp. 751-756, 1972.

[10] X. Mi, S. Zhang, H. Yu, H. Yu, M. Cong, and X. Qi, “Using a unique mirror to minimize the effect of ruling engine cosine error on grating performance," Applied Optics, vol. 57, no. 35, pp. 10146-10151, 2018.

[11] X. T. Mi, S. W. Zhang, X. D. Qi, H. L. Yu, H. Z. Yu, and Y. G. Tang, "Ruling engine using adjustable diamond and 
interferometric control for high-quality gratings and large echelles," Optics Express, vol. 27, no. 14, pp. 19448-19462, 2019.

[12] X. Li, H. Yu, X. Qi et al., “300 mm ruling engine producing gratings and echelles under interferometric control in China," Applied Optics, vol. 54, no. 7, pp. 1819-1826, 2015.

[13] Z. Li, J. Gao, H. Yang, T. Wang, and X. Wang, "Roughness reduction of large-area high-quality thick $\mathrm{Al}$ films for echelle gratings by multi-step deposition method," Optics Express, vol. 23, no. 18, pp. 23738-23747, 2015.

[14] G. R. Harrison, "The production of diffraction gratings: II the design of echelle gratings and Spectrographs1," Journal of the Optical Society of America, vol. 39, no. 7, pp. 522-528, 1949.

[15] Jirigalantu, X. Li, X. Mi, K. Liu, and Y. Tang, "Development of a parameterized mechanical model of a chisel-edge grating ruling tool," Precision Engineering, vol. 50, pp. 388-392, 2017.

[16] Z. Liu, J. Sun, and W. Shen, "Study of plowing and friction at the surfaces of plastic deformed metals," Tribology International, vol. 35, no. 8, pp. 511-522, 2002.

[17] H. Yang, Z. Li, X. Wang, Z. Shen, J. Gao, and S. Zhang, "Radial-quality uniformity investigations of large-area thick Al films," Optical Engineering, vol. 54, no. 4, Article ID 045106, 2015.

[18] H. T. Rahal, R. Awad, A. M. A. Gaber, and M. Roumie, "Superconducting and mechanical properties of the bulk $\left(\mathrm{SnO}_{2}\right)_{x}\left(\mathrm{Bi}_{1.6} \mathrm{~Pb}_{0.4}\right) \mathrm{Sr}_{2} \mathrm{Ca}_{2} \mathrm{Cu}_{3} \mathrm{O}_{10-\delta}$ prepared at different sintering times," Journal of Superconductivity and Novel Magnetism, vol. 30, no. 7, pp. 1971-1980, 2017.

[19] H. T. Rahal, R. Awad, and A. M. Abdel-Gaber, "Influence of nitrogen immersion on the mechanical properties of $(\mathrm{NiO})_{x}\left(\mathrm{Bi}_{1.6} \mathrm{~Pb}_{0.4}\right) \mathrm{Sr}_{2} \mathrm{Ca}_{2} \mathrm{Cu}_{3} \mathrm{O}_{10-\delta}$ composite," Physica B: Condensed Matter, vol. 536, pp. 803-809, 2018. 